\title{
Microstructure Analysis of Electroplated Cr-Fe Alloy Deposits on Cu Substrate
}

\author{
Che-Kuan Lin ${ }^{1,2}$, Ching-An Huang ${ }^{1}$, Wen-An Chiou ${ }^{2}$, Chao-Yu Chen ${ }^{1}$, Chin-Yu Chen ${ }^{1}$ \\ ${ }^{1 .}$ Department of Mechanical Engineering, Chang Gung University, Taoyuan, Taiwan \\ 2. NISP Lab, Nanocenter, University of Maryland, College Park, Maryland, USA
}

Since a series of health hazard toxic processes in electroplating industry, e.g., $\mathrm{Cr}^{6+}$ electroplating, has been banned under RoHS (Restriction of the Use of Certain Hazardous Substance in EEE) regulation, trivalent $\mathrm{Cr}$ electroplating has becoming one of the alternatives for $\mathrm{Cr}^{6+}$ electroplating. An eco-friendly trivalent $\mathrm{Cr}$-based electroplating bath with high efficiency has recently developed, and believed that Cr-based alloy deposits have better corrosion resistance than pure $\mathrm{Cr}$ deposits [1]. This paper reports the morphological/microstructural study of $\mathrm{Cr}-\mathrm{Fe}$ alloy deposits on $\mathrm{Cu}$ rotating cylinder electrodes (RCE) that has electroplated in an eco-friendly $\mathrm{Cr}^{3+}$ and $\mathrm{Fe}^{2+}$ based solution.

Commercial copper bars were cut/shaped as RCE with an exposure area of $2 \mathrm{~cm}^{2}$ around the cylinder. RCE surface was mechanically ground, pickled and cleaned with de-ionized water before electroplating taken place. RCE were rotated at $300 \mathrm{rpm}$ and electroplated in the electroplating bath that consisted of $0.8 \mathrm{M} \mathrm{CrCl}_{3} \cdot 6 \mathrm{H}_{2} \mathrm{O}, 0.4 \mathrm{M} \mathrm{FeSO}_{4} \cdot 7 \mathrm{H}_{2} \mathrm{O}, 0.2 \mathrm{M} \mathrm{Na}_{3} \mathrm{C}_{6} \mathrm{H}_{5} \mathrm{O}_{7} \cdot 2 \mathrm{H}_{2} \mathrm{O}, 0.4 \mathrm{M}$ urea, complexing agent and a small amount of conductive and buffer salts with the electroplating current density (EPCD) of $0.2,0.25$, or $0.3 \mathrm{Acm}^{-2}$ for 30,24 , or 20 minutes, respectively. Some of plated specimens were rapidly heated at a heating rate of $3{ }^{\circ} \mathrm{C} / \mathrm{S}$ and annealed at $500{ }^{\circ} \mathrm{C}$ for one second in the RTA (rapid thermal annealing, Model ULVAC RHL-P610CP) vacuum chamber. The Cr-Fe alloy coating/deposits on RCE were examined using a Hitachi SU-70 FEG SEM equipped with a Bruker EDS system.

EDS analysis shows the electroplated deposit/coating was Cr-Fe alloy and homogeneous throughout (Fig. 1). The Cr content in the deposits increased from 30 to $61 \mathrm{wt} \% \%$ when the EPCD increased from 0.2 to $0.3 \mathrm{Acm}^{-2}$ whereas the Fe content decreased from 63 to $27 \mathrm{wt} \%$ with the increase of EPCD (Fig. 1). Note that $\mathrm{C}$ content also increased gradually with the increase of EPCD due to the reduction mechanism. It is believed that the complex-formed $\mathrm{Cr}$ ions contain some $\mathrm{C}$ and co-deposited onto the cathode simultaneously [2]. SEM images of the as-grown Cr-Fe alloy deposits, as shown in Figure 2, reveal typical nodular (rough bumpy) morphology on the surface of RCE. Although nodular morphology were presented in all electroplating condition, the size of nodular varies with EPCD, ranging between $1 \sim 5 \mu \mathrm{m}$ in $0.2 \mathrm{Acm}^{-2}$ and $1 \sim 15 \mu \mathrm{m}$ in $0.3 \mathrm{Acm}^{-2}$. It is believed that the nodular surface of $\mathrm{Cr}-\mathrm{Fe}$ alloy deposit was resulted from high EPCD and impurities in the electroplating solution. At high magnification, it appears that the surface roughness reduces with the increase of EPCD in addition to many small pin holes surrounding nodular spots (see upper inserts in Fig. 2). Those small pin holes are believed to be caused by the escape of hydrogen bubbles during electroplating [3]. Cross-section SEM views also reveal some of residual passages created by hydrogen gas. With high EPCD the residual stress was accumulated and led to the network-like cracks on Cr-Fe alloy deposits. The width of cracks was less than $1 \mu \mathrm{m}$ in as-grown deposits, but it widened to ca. $2 \mu \mathrm{m}$ and then ca. $3 \mu \mathrm{m}$ as EPCD increased from 0.2 to 0.25 and then $0.30 \mathrm{Acm}^{-2}$, respectively (Fig 2). The number of cracks also increased with the increases of EPCD from less 5 to about 15. Thus, the width and the amount of surface cracks increase with the increases of EPCD. Cross-sectional SEM images (lower inserts in Fig. 2) depicts the thickness of Cr-Fe alloy deposits varies with EPCD, i.e., the higher the current the thicker the deposit. The thickness of Cr-Fe alloy deposits was 9.4, 13.2 and $18.9 \mu \mathrm{m}$ which were formed with EPCD at $0.2,0.25$ or $0.3 \mathrm{Acm}^{-2}$, respectively. Although the total electroplating charge could be the same (720 Coulomb), electroplating efficiency may vary with different EPCD. Thus, the $\mathrm{Cr}-\mathrm{Fe}$ alloy deposit with $0.2 \mathrm{Acm}^{-2}$ has the worst efficiency as it formed the smallest thickness and the 
largest amounts of pinholes among those deposits. During the rapid annealing, the $\mathrm{Cr}-\mathrm{Fe}$ alloy deposits and $\mathrm{Cu}$ substrate were heated $\left(500{ }^{\circ} \mathrm{C}\right)$ at the same time. Due to the moderately different coefficient of thermal expansion (CTE) between $\mathrm{Cr}-\mathrm{Fe}$ alloy $\left(6 \sim 12 \times 10^{-6}\right)$ and $\mathrm{Cu}\left(17 \sim 19 \times 10^{-6}\right)$, extra residual stress was built up at the interface between deposits and substrate. In addition, annealing also created additional thermal stress. The widening and creating more cracks in the annealed Cr-Fe alloy deposits was attributed from the release of part of residual stress (Fig. 3).

[1] Z. Zeng et al, Electrochim. Acta 52 (2006) p.1366.

[2] S. C. Kwon et al, Surface and Coatings Tech., 183 (2004) p.151.

[3] Y.B. Song, D.-T. Chin, Electrochim. Acta 48 (2002) p.349.

[4] SEM work performed at NISP Lab was partially supported by NSF-MRSEC (DMR 05-20471, Shared Experimental Facility) and UMD.

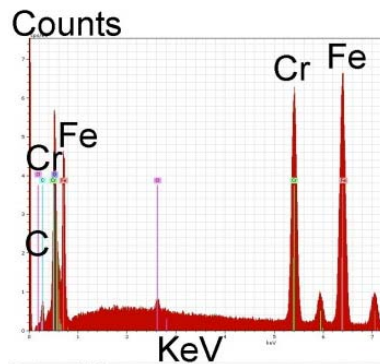

(a) $0.2 \mathrm{Acm}^{-2}$

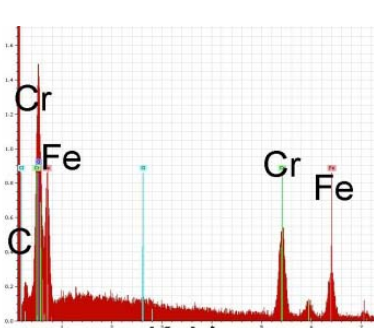

$\mathrm{KeV}$

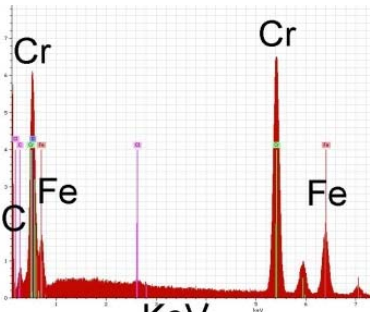

$\mathrm{KeV}$

(b) $0.25 \mathrm{Acm}^{-2}$

(c) $0.3 \mathrm{Acm}^{-2}$

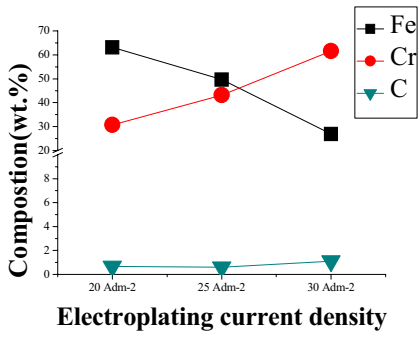

(d)

Fig. 1. EDS spectrum Cr-Fe alloy deposits formed with EPCD at (a) 0.2, (b) 0.25 and (c) $0.3 \mathrm{Acm}^{-2}$ as well as the relation between EPCD and elemental composition of Cr-Fe deposits

(a)

(b)

(c)

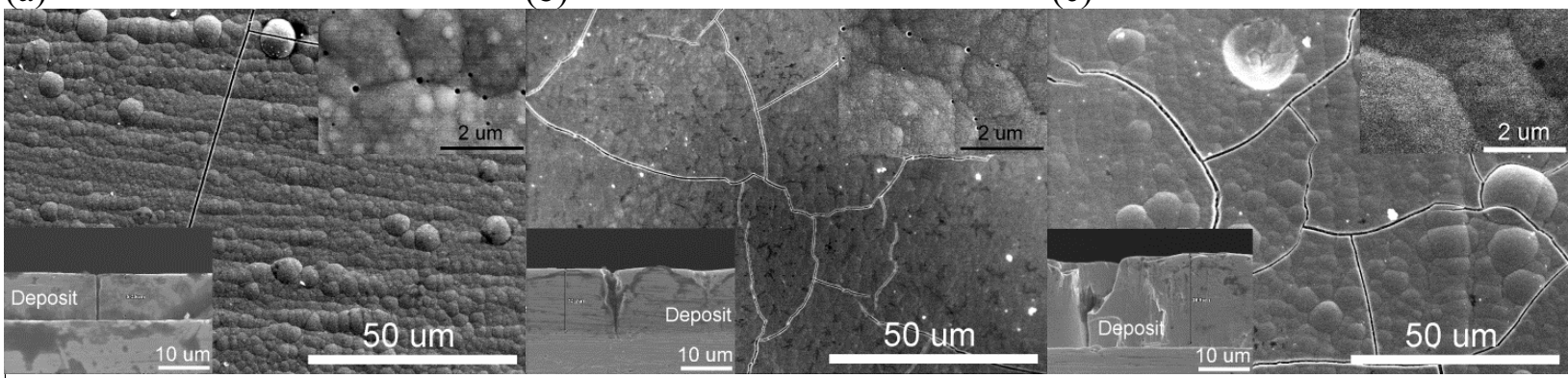

Fig. 2. SEM images showing surface morphology of as-grown Cr-Fe alloy deposit electroplated with EPCD at (a) 0.2 , (b) 0.25 and (c) $0.3 \mathrm{Acm}^{-2}$

(a)

(b)

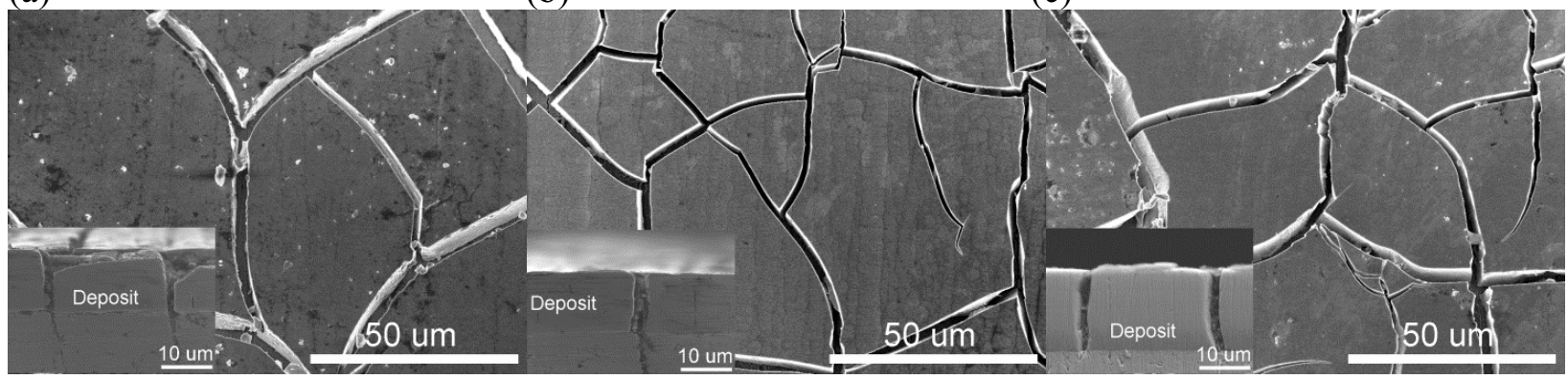

Fig. 3. SEM images revealing surface morphology of anneald $\mathrm{Cr}-\mathrm{Fe}$ alloy deposits electroplated with EPCD at (a) 0.2 , (b) 0.25 and (c) $0.3 \mathrm{Acm}^{-2}$ 